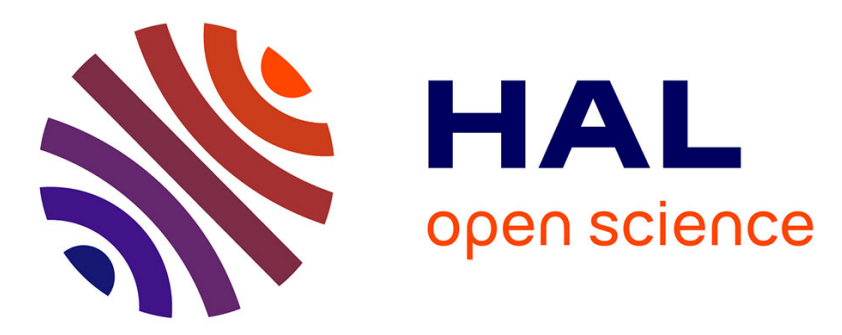

\title{
X-Ray Diffraction Study of Thermally and Stress-Induced Phase Transformations in Single Crystalline Ni-Mn-Ga Alloys \\ V. Martynov
}

\section{> To cite this version:}

V. Martynov. X-Ray Diffraction Study of Thermally and Stress-Induced Phase Transformations in Single Crystalline Ni-Mn-Ga Alloys. Journal de Physique IV Proceedings, 1995, 05 (C8), pp.C8-91C8-99. 10.1051/jp4:1995810 . jpa-00254058

\section{HAL Id: jpa-00254058 https://hal.science/jpa-00254058}

Submitted on 1 Jan 1995

HAL is a multi-disciplinary open access archive for the deposit and dissemination of scientific research documents, whether they are published or not. The documents may come from teaching and research institutions in France or abroad, or from public or private research centers.
L'archive ouverte pluridisciplinaire HAL, est destinée au dépôt et à la diffusion de documents scientifiques de niveau recherche, publiés ou non, émanant des établissements d'enseignement et de recherche français ou étrangers, des laboratoires publics ou privés. 


\title{
X-Ray Diffraction Study of Thermally and Stress-Induced Phase Transformations in Single Crystalline Ni-Mn-Ga Alloys
}

\author{
V.V. Martynov(1) \\ Institute of Physics of Metals National Academy of Sciences of Ukraine, Kiev, Ukraine
}

\begin{abstract}
Using in-situ single crystal X-ray diffraction methods, thermally- and stress-induced crystal structure evolution was investigated in two Ni-Mn-Ga Heusler-type alloys. For the 51at.\%Ni-24at.\%Mn-25at.\%Ga alloy it was found that application of external stress in a temperature range $\sim 20{ }^{\circ} \mathrm{C}$ above the $\mathrm{M}_{\mathrm{s}}$ at first causes intensity changes of $\mathrm{X}$-ray diffuse scattering peaks in $\beta$-phase. Further stressing results in stress-induced phase transformations and under the appropriate conditions three successive martensitic transformations (one is parent-tomartensite and two are martensite-to-martensite transformations) can be stress induced. Of these only the parent-tomartensite transformation can be thermally-induced. Two successive structural transformations (thermally-induced parent-to-martensite and stress-induced martensite-to-martensite transformations) were found in 52at.\% $\mathrm{Ni}$ 25at.\%Mn-23at.\%Ga alloy. Crystal structure, lattice parameters, type of modulation, and the length of modulation period for all martensites were identified
\end{abstract}

\section{INTRODUCTION}

As was found in the earlier investigation, the nearly stoichiometric $\mathrm{Ni}_{2} \mathrm{MnGa}$ Heusler-type alloy exhibits martensitic structural phase transition on cooling below the Curie temperature [1]. Tetragonal distortion of an ordered b.c.c. (L2 $2_{1}$ lattice of the parent phase taking place during this transformation results in the formation of a martensite lattice having parameters $a=b=0.59 \mathrm{~nm}, c=0.554 \mathrm{~nm}$. Extra diffraction maxima observed at X-ray diffraction patterns of the martensite were interpreted as due to periodically arranged stacking faults along the $c$ axis of martensite [1]. In more recent investigations [2] it was shown that martensite identical to thermally-induced martensite can be stress-induced by compression of single crystals along a $<100>$ direction. The presence of extra spots at X-ray diffraction patterns of martensite was rationalized in terms of lattice modulation by static waves of shear displacements having the wave vector normal to one of two (110), (110) planes and wave length equal to $5(110)$ interlayer spacing. Later two more successive stress-induced transformations in Ni-Mn-Ga single crystals were reported for compression along $<110>$ and tension along $<100>$ directions [3-6].

In this paper we briefly review our former experimental results and also report recent findings in changes of X-ray diffuse scattering in uniaxially compressed $\beta$ phase, the X-ray diffraction study of thermally and stress-induced phase transitions in 52at.\%Ni-25at.\%Mn-23at.\%Ga single crystals.

\section{MATERIALS AND EXPERIMENTAL TECHNIQUE}

Alloys with the nominal compositions 51 at.\%Ni-24at.\%Mn-25at.\%Ga (alloy 1) and 52at.\%Ni-25at.\%Mn23at.\%Ga (alloy 2) were melted in a high frequency induction furnace under argon atmosphere and cast into a cylindrical mould $15 \mathrm{~mm}$ in diameter. Single crystals were prepared from the ingots using the conventional Bridgman's method. For the alloys used in the present experiment transformation temperatures were established to be $M_{s}=293 \mathrm{~K}, M_{f}=273 \mathrm{~K}, A_{s}=278 \mathrm{~K}, A_{\mathrm{f}}=298 \mathrm{~K}$ for alloy 1 and $M_{s}=333 \mathrm{~K}$,

(1) Present address: Ti-Ni Alloy Company, 1621 Neptune drive, San Leandro, CA 94577, U.S.A. 
$M_{\mathrm{f}}=273 \mathrm{~K}, \mathrm{~A}_{\mathrm{s}}=283 \mathrm{~K}, \mathrm{~A}_{\mathrm{f}}=345 \mathrm{~K}$ for alloy 2 with the scattering $\pm 5 \mathrm{~K}$ due to composition inhomogeneity along the single crystals. Typical resistivity vs. temperature curves for the alloys used are shown in fig. 1 .

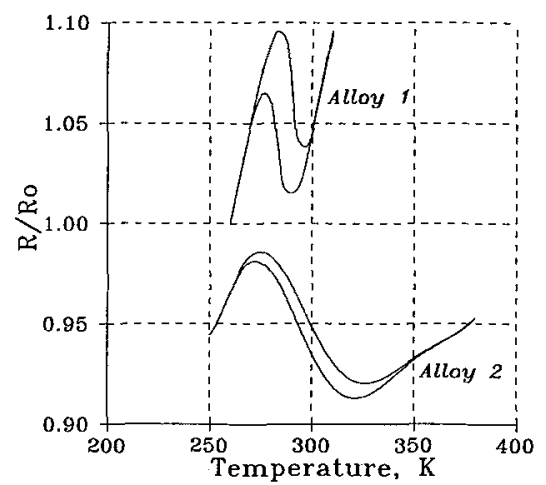

Figure 1: Resistivity changes due to thermally-induced martensite transition.

Bars $2 \times 2 \mathrm{~mm}$ in cross section were spark cut from the single crystals along $\langle 100\rangle$ and $<110\rangle$ directions. Specimens to be used for X-ray structural analysis, uniaxial tension, and compression experiments were ground from these bars, using a diamond grinding wheel. All specimens were of the same "dumbbell "shape. Specimens with a gauge length of $10 \mathrm{~mm}$ used for tension experiments were ground from the entire single crystalline bar and subsequently electropolished in an electrolyte consisting of nitric and acetic acids in a ratio 1:1 by volume. The final diameters of the "heads" and the main part of the specimen were equal to $1.0 \mathrm{~mm}$ and $0.5 \mathrm{~mm}$ respectively. Specimens for compression experiments (gauge length 2-3 mm) were made of a combination of electropolished single crystalline rod $0.5-0.7 \mathrm{~mm}$ in diameter and cylindrical brass "heads "soldered to it by a tin solder.

Mechanical tests were performed using a ZM-40 mechanical testing machine, adjusted for compression and tension of small specimens. For temperature stabilization purpose specimens during mechanical tests were dipped in a Dewar vessel filled with water or petrol heated or cooled to a predetermined temperature. The X-ray rotating and oscillating single-crystal methods with photo registration performed at room temperature were used for the crystal structure analysis. Diffuse scattering data acquisition was conducted by using a Rigaku four circle diffractometer (AFC-5) with a rotating anode X-ray generator (RU-200). Special jigs were used for in situ tension and compression of specimens in the X-ray chamber and the diffractometer.

\section{RESULTS AND DISCUSSION}

Lattice parameters of high-temperature L $2_{1}$ ordered $\beta_{3}$-phase measured at room temperature for alloy 1 were found to be: $a=b=c=0.582 \mathrm{~nm}$ and are in good agreement with the values reported in [1] (due to the closeness of atomic scattering factors for constituent elements of Ni-Mn-Ga alloy only diffraction spots of A2 crystal structure were resolved at the X-ray diffraction patterns). As has been the case with electron microscopy observations, the main reflections of $\beta_{3}$-phase are broadened and exhibit intensive diffuse streaks along $<110>*$ directions.

\subsection{Crystal structure of thermally-induced martensites.}

Spontaneous phase transition during cooling results in the formation of many martensite variants within the same single crystal. However, by using a low cooling rate with small temperature gradients along the single crystal or slow cooling with a constant tension or compression stress ( $\sigma \sim 10 \mathrm{MPa})$ applied to the specimen it is possible to obtain relatively large martensite crystals which are suitable for structural analysis. 


\subsubsection{Crystal Structure of Thermally-induced Martensite in Alloy 1}

From the analysis of the most intensive X-ray diffraction spots the crystal structure of martensite formed during cooling can be interpreted in terms of a body centered tetragonal lattice with its main axes parallel to the cubic axes of $\beta_{3}$ phase. Calculated lattice parameters $a=b=0.590 \mathrm{~nm}, c=0.554 \mathrm{~nm}$ (regarding the L2 $2_{1}$ ordering of high temperature phase, measured values should be doubled) are in good agreement with those reported for martensite in [1].

Along with the main strong spots, additional relatively weak diffraction maxima were also observed at oscillation diffraction patterns. These spots are situated along reciprocal lattice rows which are parallel to one of two $\langle 110\rangle^{*}$ directions and divide the distance between the main spots into 5 equal parts. If one denotes the intensity of the main diffraction spots as the "strong" (S) and additional ones as "mediate" (M) and "weak" (W) the intensity distribution along $<110>*$ direction between the main spots can be written as follows: $S-M-W-W-M-S$. Absolute intensities of additional spots depend on the indices of the particular reciprocal row chosen; however, the above mentioned qualitative intensity distribution is valid for all of them.

From the analysis of X-ray patterns of martensite it was concluded that additional maxima are the result of periodical shuffling (modulation) along the (110)[110] system of martensite in such a way that each 5-th (110) plane remains in its original position. Thus, the shear modulation of the martensite lattice may be considered as superimposed waves of static displacements having propagation vectors $\mathbf{k} \|[110]^{*}$ and polarization vectors $\mathbf{e} \|[1 \overline{1} 0]^{*}$.

If we write the displacement $\Delta_{\mathrm{j}}$ of the $\mathrm{j}$-th plane from the regular position in shuffling direction as:

$$
\Delta_{\mathrm{j}}=\operatorname{ASin} 2 \pi \mathrm{j} / \mathrm{L}+\mathrm{BSin} 4 \pi \mathrm{j} / L+C \operatorname{Sin} 6 \pi \mathrm{j} / L \text {, }
$$

where $\mathrm{L}$ is the length of the modulation period, constants $\mathrm{A}, \mathrm{B}$ and $\mathrm{C}$ could be selected using the least discrepancy between the experimentally measured intensities of the main and additional spots and calculated $|\mathrm{F}|^{2}$ values as the criterion. Corresponding constants calculated for the thermally induced 5layers martensite $(\mathrm{L}=5)$ are: $\mathrm{A}=-0.06, \mathrm{~B}=0.002, \mathrm{C}=-0.007$. Measured and calculated intensities along a particular [110] direction are listed in the table 1.

Table 1: Measured (I) and calculated $\left(|\mathrm{F}|^{2}\right)$ intensities for 5-layers martensite.

\begin{tabular}{ccc}
\hline hkl & I & $|\mathrm{F}|^{2}$ \\
\hline 110 & 74 & 74.12 \\
$\mathrm{M}$ & 14 & 13.98 \\
$\mathrm{~W}$ & $<1$ & $<0.01$ \\
$\mathrm{~W}$ & $<1$ & 1.08 \\
$\mathrm{M}$ & 11 & 10.82 \\
200 & 74 & 74.12 \\
\hline
\end{tabular}

\subsubsection{Crystal Structure of Thermally-induced Martensite in Alloy 2}

The crystal structure of thermally-induced martensite in alloy 2 differs from the previous one both in symmetry and in modulation period length. Additional diffraction spots, which also lie in the $<110\rangle$ direction, now divide the distance between the main maxima into 7 equal parts $(\mathrm{L}=7)$. According to the main diffraction spots, the crystal lattice of this martensite phase may be regarded as body centered monoclinic. Taking into account atomic ordering, lattice parameters were calculated to be: $a=0.614 \mathrm{~nm}$, $b=0.578 \mathrm{~nm}, c=0.551 \mathrm{~nm}, \gamma=90.5^{\circ}$. Using the above described approach for evaluation of the modulation parameters one can find that the experimentally observed intensity distribution for 7-layer modulation can be described by $\mathrm{A}=0.083, \mathrm{~B}=-0.027, \mathrm{C}=0$. Measured and calculated intensities for $20 l$ 
relrow (in the coordinate system where the $c$-axis is chosen along the modulation direction) are listed in the table 2 .

Table 2: Measured (I) and calculated $\left(|\mathrm{F}|^{2}\right)$ intensities for 7-layers martensite.

\begin{tabular}{ccc}
\hline hkl & $\mathrm{I}_{\mathrm{m}}$ & $|\mathrm{F}|^{2}$ \\
\hline 200 & 51 & 52.40 \\
202 & 23 & 27.34 \\
204 & $<1$ & $\sim 0.01$ \\
206 & $<1$ & 0.07 \\
208 & $\sim 1$ & 0.96 \\
2010 & 7 & 6.25 \\
2012 & 18 & 12.96 \\
\hline
\end{tabular}

\subsection{Stress-induced transformations}

As has been established in recent years, martensitic transformations in many b.c.c. $\beta$ phase alloys are associated with the lattice instability which, when approaching the transformation temperature manifests itself in typical premonitory effects, namely, elastic constants and phonon dispersion curves anomalies, $\mathrm{X}$-ray and electron diffuse scattering. In particular, in $\mathrm{Ni}-\mathrm{Al}$ alloys [6] intensive diffuse scattering of electrons along $<110>$ directions and also remarkable softening of the transverse acoustic $\mathrm{TA}_{2}$ phonon branch observed around $\mathbf{q} 1 / 7$ [110], are correlated with 7-layer (5.2) martensite, formed in the alloy on further cooling [7]. As it was reported in [8] intensity of X-ray diffuse scattering in $\mathrm{Ni}_{2} \mathrm{MnGa}$ is also strongly temperature dependent and peaks around $q \sim 1 / 6[110]$ begin to appear at a temperature about 80

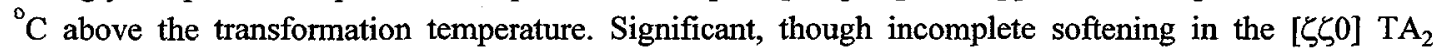
phonon branch at a wave vector $\zeta_{0} \sim 0.33$ for $\mathrm{Ni}_{2} \mathrm{MnGa}$ was also reported in [9]. Apart from the thermally induced martensitic transformation, in many $\beta$-phase alloys transformation can also be induced by application of external stresses; however, only a limited number of publications concerning stress-induced premonitory effects are available at present $[10,11]$.

\subsubsection{Effect of Uniaxial Compression on X-ray Diffuse Scattering}

$\mathrm{X}$-ray diffuse scattering measurements in single crystals of alloy 1 were performed for stress-free and uniaxially compressed states at the temperature above the $M_{S}$ point. Diffuse scattering was measured along $<110>$ directions with the interval equal to $1 / 200$ of the distance between the main Bragg reflections of $\{400\}$ and $\{220\}$ type.

A specimen mounted in the jig was compressed along $<100>$ direction until the first plate of stress induced martensite appeared (an optical microscope was used for visual control). For certain specimen the maximum possible stress applied was $\sim 35 \mathrm{MPa}$ given an ambient temperature of $\sim 22{ }^{\circ} \mathrm{C}$. Next the specimen still mounted in a jig was installed in a diffractometer and diffuse scattering in $\beta_{3}$ phase along different $\langle 110\rangle$ directions was measured. To minimize the effect of geometric factors on the intensity measurements, after measurements in the compressed state were finished, the specimen was released (or compressed further to obtain a single crystal of stress induced martensite) without taking the jig out of the diffractometer.

When diffraction vector $\mathbf{g}$ was parallel to $\langle 110\rangle$ directions in which diffuse scattering was measured, no anomalies were observed. Otherwise two additional peaks appeared in the vicinity of the Bragg reflections (fig. 2). The maximum intensity of these additional peaks is $\sim 0.004$ of the main maxima. Each has a halfwidth of $\sim 0.12 \mathrm{~nm}^{-1}$ and each is separated from the main maximum by about 0.160 of the distance between Bragg reflections $(\sim 1 / 6[110])$. These peaks in the real space correspond to transverse 
waves of atomic displacements along $<110>$ directions (peaks of diffuse scattering weren't observed for $\mathrm{g} \| \mathbf{q})$ with the wave length equal to $\sim 6$ translation periods.

The effect of applied stress on the intensity of the additional peaks was different depending on the angle the particular $<110>$ direction forms with the compression axis. For two $<110>$ directions which are normal to the compression axis, the intensity of diffuse scattering peaks decreases slightly after releasing the stress (fig. 2a). For the remaining four $\langle 110\rangle$ directions, which form a $45^{\circ}$ angle with the compression axis, intensities of additional maxima remain almost unchanged and even very weak tendencies for the diffuse scattering peaks to increase after the release of stress could be deduced from the experimental data.

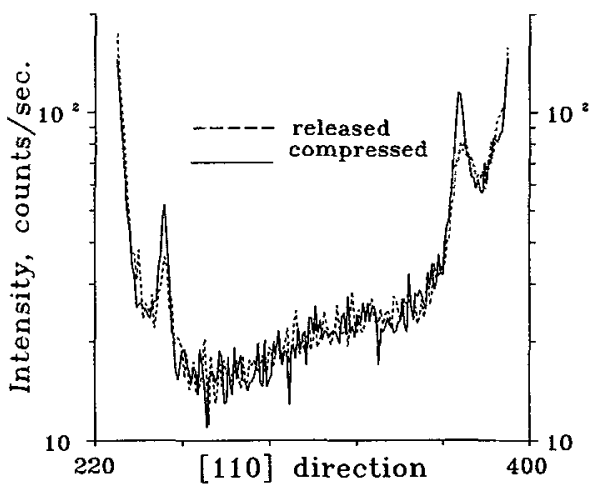

a)

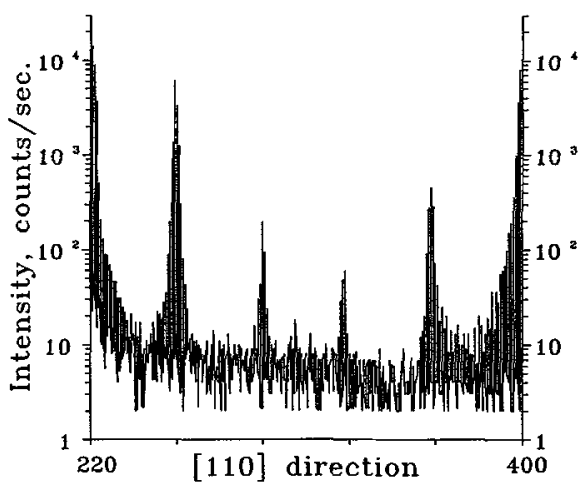

b)

Figure 2: Diffuse scattering along [110] direction in $\beta_{3}$ phase (a) and stress-induced martensite (b). Compression axis [001].

On further stressing $\beta_{3}$ phase transforms to stress-induced martensite. One variant of martensite with its $c$ axis parallel to the compression direction is formed. Broadening and splitting of martensite reflections due to bending of the specimen make accurate lattice parameter determination difficult, however measured values ( $a=0.59 \mathrm{~nm}, b=0.589 \mathrm{~nm}, c=0.559 \mathrm{~nm}$ ) are in reasonable agreement with those measured for thermally induced 5-layer martensite. Diffuse scattering intensity measurements along the [110] direction reveal four additional peaks between the main Bragg reflections (fig. 2b), implying 5layers transverse modulation.

\subsubsection{Deformation Behavior and Related Stress-induced Martensite Transformation for Uniaxial Compression}

All specimens used for compression experiments were made of alloy 1 . For compression along the $<100>$ direction the maximum specimen's contraction value, corresponding to formation of martensite in the whole specimen's volume was about $5 \%$. Depending on the deformation temperature, inelastic contraction achieved during loading either remained stable up to subsequent heating or superelasticity loops (fig. 3 a) were observed. Stress necessary for stress-induced martensite formation in the temperature range above $M_{s}$ linearly follows the deformation temperature with the rate $d \sigma / d T=2.5 \mathrm{MPaK}^{-1}$. Enthalpy change, calculated from the Clausius-Clapeyron-like equation using experimentally obtained $\mathrm{d} \sigma / \mathrm{dT}$ is in satisfactory agreement with the calorimetric data for thermally-induced transformation.

As follows from the analysis of $\mathrm{X}$-ray diffraction patterns, compression along $<100\rangle$ direction results in the formation of a single crystal of martensite with its $c$ axis nearly parallel to the compression direction. Crystal structure of stress-induced martensite is identical to thermally-induced crystal structure reported above. The amount of recoverable deformation for the particular stress-induced martensite variant formed is in a good agreement with the lattice deformation accompanying structural phase transition.

In contrast with the previous case, a typical stress-strain (s-s) curve for compression along $\langle 110\rangle$ direction consists of more than one deformation stage having corresponding plateaus on loading and 
unloading branches. The number of stages depends on the deformation temperature and three distinct plateaus were observed at the loading branch at $279 \mathrm{~K}$, while unloading one consists only of two plateaus (fig. $3 \mathrm{~b}$ ). (The dashed line indicates the beginning of the s-s curve for the second and following cycles.) For this particular deformation temperature contraction corresponding to the first deformation stage in the first loading cycle is stable upon unloading.

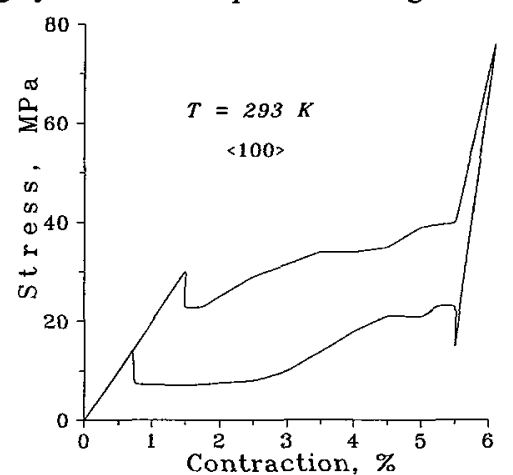

a)

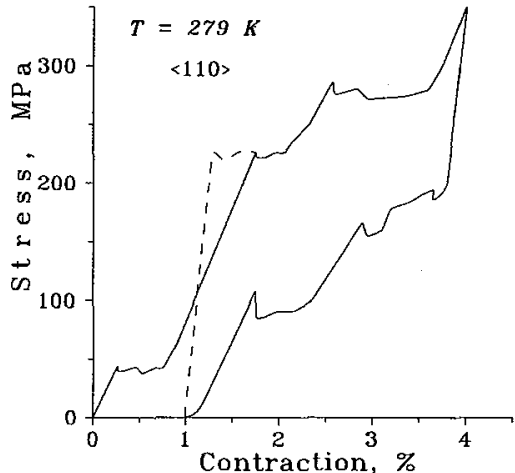

b)

Figure 3: Stress-strain curves for compression along $<100>$ (a) and $<110>$ (b) directions.

Observation of the surface of polished specimens during loading shows that at the beginning of each deformation stage few thin plates crossing the whole cross section of specimen initially arise. As the deformation proceeds, already existing plates become thicker and new plates appear in different parts of the specimen. At the end of a corresponding stage all of them coalesce together changing the appearance over the whole specimen length. Reverse processes take place upon unloading.

Every stage of the loading branch of the s-s curve corresponds to the formation of a particular martensite crystal structure. Stress-induced martensite corresponding to the first plateau has essentially the same crystal structure as martensite formed during compression along the $<100>$ direction.

Further increase of the load results in the formation of a new crystal structure. Now additional spots, which are still situated along $\langle 110\rangle^{*}$ direction, divide the distance between the main spots of martensite in 7 equal parts. According to the most intensive diffraction spots the crystal lattice of this stress-induced phase can be regarded as body centered monoclinic with its $a$ axis normal to the compression direction. Lattice parameters were calculated to be $a=0.612 \mathrm{~nm}, b=0.578 \mathrm{~nm}, c=0.554 \mathrm{~nm}, \gamma=90.5^{0}$ and are very close ${ }^{1}$ to those for thermally induced martensite in alloy 2 .

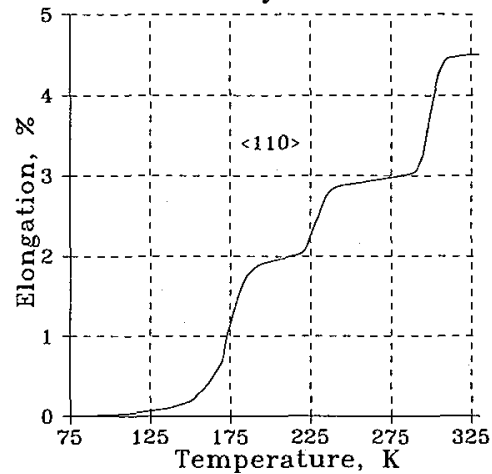

Figure 4: Shape recovery during heating after compression at $77 \mathrm{~K}$.

\footnotetext{
${ }^{1}$ In the previous publications [3-5] this crysttal structure was refed as orthorhombic having $\alpha=\beta=\gamma=90^{\circ}$
} 
After the monoclinic martensite was formed throughout the entire volume of the specimen further increase of applied stress results in one more stress-induced structural transformation. X-ray patterns taken from the single crystal formed show a disappearance of spots due to shuffling modulation and indicate that new b.c.t. crystal structure is formed. Lattice parameters of this phase were established to be $a=b=0.552 \mathrm{~nm}, c=0.644 \mathrm{~nm}, c$ axis is normal to the compression direction. In contrast with 5-layers b.c.t. martensite, newly formed martensite has a $c / a$ ratio greater than unity.

The stress level, necessary for inducing martensite-to-martensite transformations decreases with the lowering of the deformation temperature. Successively formed stress-induced martensites become stable after unloading below $233 \mathrm{~K}$ and $193 \mathrm{~K}$ respectively; however, during heating complete recovery of initial dimensions was always observed. Fig. 4 shows a typical length recovery curve for a specimen compressed at $77 \mathrm{~K}$.

\subsubsection{Deformation Behavior and Related Stress-induced Martensite Transformation for Uniaxial Tension}

Because of the very high brittleness of the $\mathrm{Ni}_{2} \mathrm{MnGa}$ intermetallic compound, tensile experiments were confined to a much lower applied stress level than that for compression. (In our experiments maximum applied stress before failure hardly exceeded $\sim 100 \mathrm{MPa}$.) This limitation in turn imposed a restriction on the temperature range for observation of a stress-induced martensite formation. Usually this range was $293-77 \mathrm{~K}$.

A typical s-s curve for a specimen with $<100>$ longitudinal axis made of alloy $\mathbf{1}$ is shown at the fig. 5 .

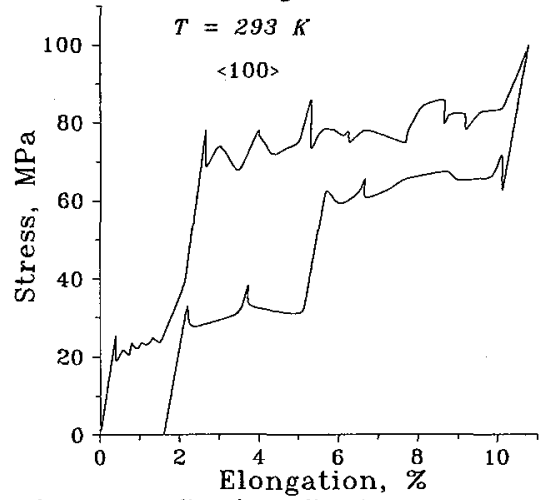

Figure 5: Stress-strain curve for tension along $<100>$ direction. Alloy 1.

With respect to tension, s-s curves along $<100>$ axis are qualitatively very close to those obtained for compression along $\langle 110\rangle$ direction. Although the crystal structure of stress-induced martensites at corresponding deformation stages is the same as for compression, larger deformations observed resulted from different variants of martensite stress-induced in compression and tension. One can also see that significantly lower stresses are required in tension for inducing a corresponding phase transition.

In contrast to the specimens prepared from alloy $\mathbf{1}$, specimens made of alloy 2 at room temperature consist mainly of thermally-induced martensite (see fig. 1). This means that in the temperature range 293$77 \mathrm{~K}$ loading starts from the multivariant martensite unless preliminary deformation had been applied to obtain a single crystal of thermally-induced martensite. Fig. 6 shows deformation behavior at $285 \mathrm{~K}$ for specimens cut in $\langle 110\rangle$ and $\langle 100\rangle$ directions (referring to $\beta_{3}$ phase).

As with X-ray analysis, in both cases a single crystal of the same 7-layer martensite is formed at the end of deformation. Particular variants of a martensite single crystal are governed by the maximum elongation for a given longitudinal axis and for $\langle 110\rangle$ and $\langle 100\rangle$ tensile axes direction martensite's orientations are [110] and [100] correspondingly. Maximum deformation calculated for a given variant from the difference in the lattice parameters for $\beta_{3}$ phase and monoclinic martensite correlates well with the measured elongation. 


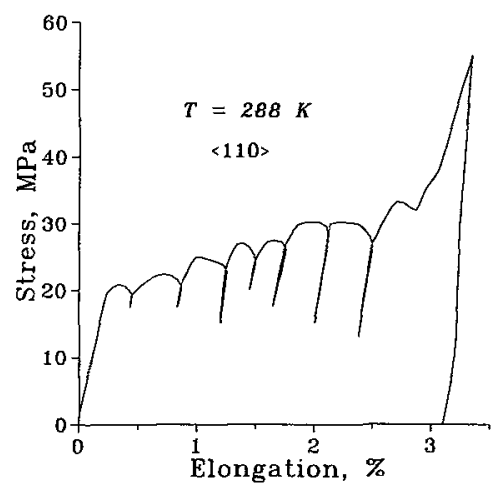

a)

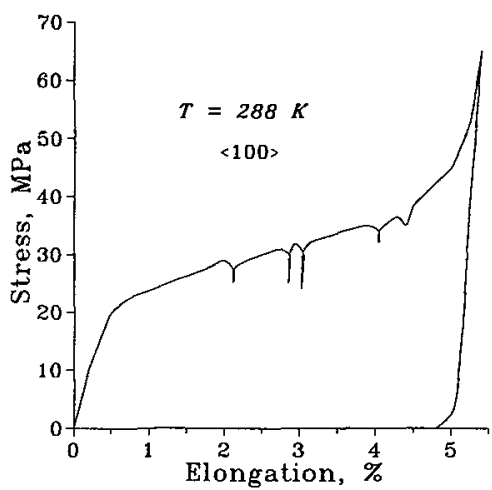

b)

Figure 6: Stress-strain curves for tension along $<110>$ (a) and $<100>$ (b) directions. Alloy 2.

Lowering of the deformation temperature results in reduction of the required stress level for martensite-to-martensite stress-induced transformation and below $273 \mathrm{~K}$ this transformation was observed in specimens with $\langle 100\rangle$ longitudinal axis. In specimens cut along $\langle 110\rangle$ direction martensite-tomartensite transformation was not observed at any deformation temperature up to $77 \mathrm{~K}$.

The stress-strain curve for a specimen with $<100>$ longitudinal axis obtained at $77 \mathrm{~K}$ is shown at fig. 7 a. Single crystal of monoclinic martensite was previously formed by deformation at $285 \mathrm{~K}$.

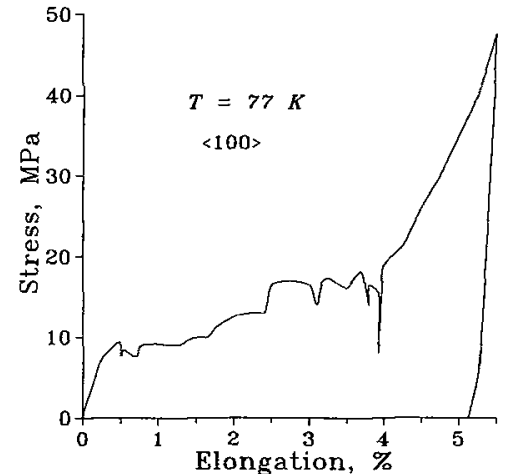

a)

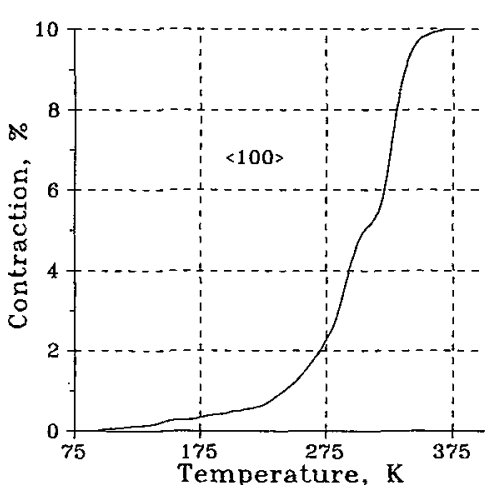

b)

Figure 7: Stress-strain curve for tension along $<100>$ direction at $77 \mathrm{~K}$ (a) and length recovery during heating after elongation at $77 \mathrm{~K}$ (b) for alloy 2

Although the stress level which is necessary for inducing martensite-to-martensite transformation at room temperature in alloy 2 is higher than the strength of $\mathrm{Ni}_{2} \mathrm{MnGa}$ single crystal, it is possible by keeping the specimen under tension to retain at room temperature the martensite which was stressinduced at lower temperatures. In this way the crystal structure of martensite was analyzed and it was found that it is identical to the crystal structure of the third martensite (tetragonal, $c / a>1$ ) stress-induced in alloy 1. Deformation calculated from the difference in lattice parameters for monoclinic and tetragonal $(c / a>1)$ martensites is in good agreement with observed (fig 7a) elongation.

Once formed martensite stress-induced at subzero temperatures become stable and does not disappear upon unloading (see fig. 7a). However, during heating complete recovery of initial length was always observed (fig. 7b). Two stages on the shape recovery curve correspond to two phase transitions. 


\section{SUMMARY}

Using single crystal X-ray diffraction methods three successive structural phase transitions were found in 51 at.\%Ni-24at.\%Mn-25at.\%Ga alloy (1) and two phase transitions were identified in 52at.\%Ni25at.\%Mn-23at.\%Ga alloy (2). The crystal structure of all phases was identified and lattice parameters were measured. Extra reflection along $\langle 110\rangle *$ relrows for two martensites were interpreted as due to shuffling modulation along (110) [110] system in the real space.

For both alloys the parent to martensite phase transitions can be either thermally induced by cooling below the transition temperature or stress-induced in a temperature range above it by compression or tension in any direction. For martensite-to-martensite phase transitions to be observed, tension along $<100>$ axis or compression along $<110>$ direction has to be applied in the temperature range $293-77 \mathrm{~K}$.

Compression of $\beta$ phase (alloy 1) along $<100>$ axis in the stress range below the critical stress necessary for creating stress-induced martensite, results in changes of X-ray diffuse scattering. It was observed that for $<110>$ directions normal to the compression axis intensity of peaks around $\mathbf{q} \sim 1 / 6$ [110] increased with increasing applied stress.

\section{Acknowledgments}

The above study was partly supported by Japan Society for Promotion of Science (JSPS).

\section{References}

[1] Webster P.J., Ziebeck K.R. A. Town S.L., Peak M.S., Philos. Mag. 49 (1984) 295-310.

[2] Zasimchuk I.K., Kokorin V.V., Martynov V. V., Tkachenko A. V., Chernenko V. A., Fiz. Met. Metalloved. (1990) N6 110-114.

[3] Kokorin V.V., Martynov V. V., Fiz. Met. Metalloved. (1991) N9 106-113.

[4] Kokorin V.V., Martynov V. V., Chernenko V. A., Scripta Met. 26 (1992) 175-177.

[5] Martynov V. V. and Kokorin V. V., J. Phys. III France 2 (1992) 739-749.

[6] Shapiro S.H., Larese J.Z., Noda Y., Moss S.C., Tanner L.E., Phys. Rev. Letters 57 (1986) 3199-3202.

[7] Martynov V.V., Enami K., Khandros L.G., Tkachenko A.V., Nenno S., Scripta Met. 17 (1983) 11731176.

[8] Fritsch G., Kokorin V.V., Kampf A., J.Phys.F., Condens. Matter. 6 (1994) L107-L110.

[9] Zheludev A., Shapiro S.M., Wochuer P., Phys. Rev. B 51, (1995) 11310.

[10] Morii Y., Iizumi M., J.Phys. Soc. Japan 54 (1985) 2948-2954.

[11] Nagasawa A., Kuwabara A., Morii Y., Fuchizaki K., Funahashi S., Mater. Trans. JIM 33 (1992) 203207. 